\title{
Akutdiagnostik von Sportverletzungen
}

\author{
Björn H. Drews
}

\section{Zusammenfassung}

Zur Einleitung einer effektiven und zeitnahen Therapie nach Sportverletzungen ist ebenfalls eine zeitnahe effektive Diagnostik erforderlich. Aus der Anamnese und klinischen Untersuchung leiten sich Dringlichkeit und Reihenfolge der weiteren apparativen Diagnostik ab. Die Sonografie stellt hier aufgrund der vornehmlich weichteiligen Verletzungen den Grundstein dar. Hiermit können bereits viele Diagnosen gestellt oder ausgeschlossen werden. Für eine weiterführende Kernspintomografie ist anhand der klinisch gestellten Verdachtsdiagnose der Zeitrahmen zu setzen. Während einige Diagnosen mit Verzögerung bildmorphologisch gesichert werden können, brauchen andere eine unmittelbare MRT zur Einleitung der weiteren Therapie und Erreichung eines optimalen Outcomes.

\section{Acute Diagnostic Testing in Sporting Injuries}

Early and efficient diagnostic testing is essential for early and target-oriented therapy in sporting injuries. The priority and sequence of diagnostic methods depend on patient history and physical examination. As soft tissue injuries are very common in this type of injury, sonography is the basic diagnostic instrument. Many diagnoses can be confirmed or eliminated with this tool. For further diagnostic testing with MRI, a time frame has to be set, depending on the suspected diagnosis. For some injuries, delayed verification by MRI can be accepted while, for others, early MRI is essential for therapy initiation and to assure an optimal clinical outcome.

\section{Einleitung}

Der Bereich der akuten Sportverletzungen umfasst Verletzungen der Muskulatur, Sehnen und Ligamente sowie auch der Knochen. Beim Hochleistungssportler ist die frühzeitige Diagnosestellung der entscheidende Faktor zur zeitnahen Therapieeinleitung und somit raschen Wiederherstellung der vollen Leistungsund Wettkampffähigkeit. Aber auch beim Hobbysportler können verzögerte Verläufe mit später endgültiger Diagnosestellung den Heilungsverlauf verzögern, das Outcome verschlechtern und die Arbeitsunfähigkeit bzw. Sportunfähigkeit verlängern.

OP-JOURNAL 2016; 32: 20-23

(C) Georg Thieme Verlag KG Stuttgart · New York DOI http://dx.doi.org/10.1055/s-0042-106749

\section{Klinische Untersuchung}

Die klinische Diagnostik gehört trotz Vormarsch und breiten Verfügbarkeit apparativer Diagnostika weiterhin zu den wichtigsten Werkzeugen des Orthopäden/Unfallchirurgen.

Hier können bereits, insbesondere im Bereich der Gelenkverletzungen, Verdachtsdiagnosen mit hoher Sensitivität gestellt werden (Abb. 1). Aus dem klinischen Verdacht ergibt sich dann der weitere apparative diagnostische Algorithmus und dessen Zeitrahmen. So kann beim Verdacht auf eine vordere Kreuzbandruptur eine Wartezeit von Tagen bis wenigen Wochen bis zum MRT in Kauf genommen werden, während bei Verdacht auf eine hintere Kreuzbandruptur oder eine Patellaluxation eine Diagnosesicherung innerhalb weniger Tage erzwungen werden sollte, um eine adäquate Therapie zeitnah einzuleiten.

\section{Sonografie}

Somit ist bei jedem Patienten nach erlittener Sportverletzung unabhängig vom Leistungslevel eine zeitnahe Diagnosestellung wichtig, um unnötig lange Ausfallzeiten auf sportlichem und beruflichem Gebiet zu verhindern.

\section{Diagnostische Verfahren}

Zur Sicherung der Diagnose stehen unterschiedliche diagnostische Mittel zur Verfügung, welche abhängig von der Verdachtsdiagnose unmittelbar oder erst im Intervall zur Anwendung kommen sollten. Die Einhaltung eines diagnostischen Algorithmus beschleunigt den diagnostischen Prozess, spart Zeit und Kosten und reduziert die Anwendung unnötiger diagnostischer Maßnahmen.
Die Ultraschalldiagnostik stellt mit die 1. Instanz der apparativen Diagnostik dar. Im Bereich der Muskel-, Sehnenund peripheren Bandverletzungen ist sie das wichtigste primäre Tool.

In der Hand des geübten Arztes lässt sich hierdurch bereits häufig die Diagnose abschließend sichern oder Verdachtsdiagnosen ausschließen. So kann z. B. bei der Distorsion des oberen Sprunggelenks, welche mit 5\% eine der häufigsten Verletzungen in der chirurgischen Notaufnahme darstellt [15], die Ruptur des medialen [6] und lateralen Kollateralbandapparats $[11,13]$ sowie auch des ventralen Syndesmosenanteils (AITFL) [12] mit hoher Spezifität und Sensitivität detektiert werden. (Abb. 2 und 3). Auch bei Sehnenrupturen wie der Achillessehnenruptur [14,17], Quadrizepssehnenruptur $[4,7,10]$ oder distalen Bizepssehnenruptur [2] lässt die Sonografie eine sichere und schnelle Diagnosestellung 


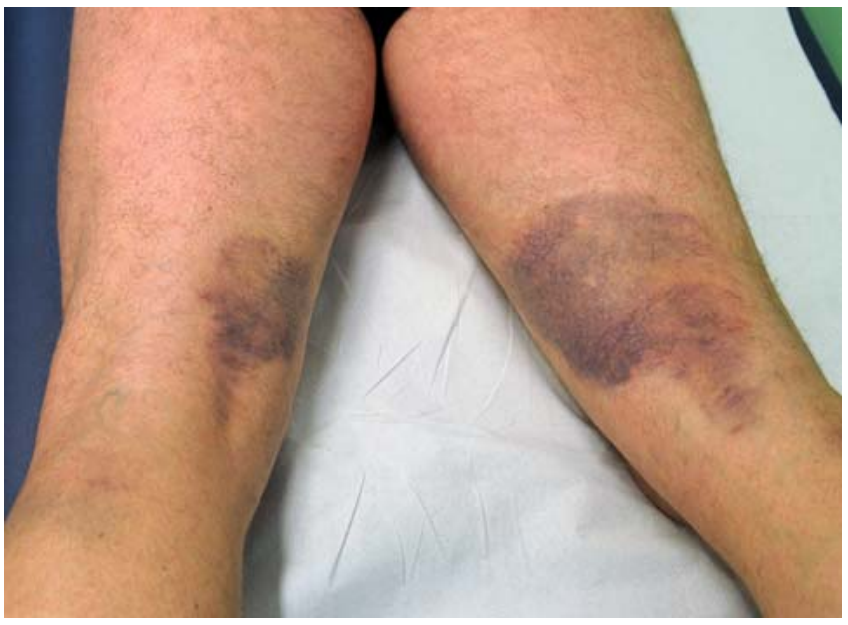

zu. Eine weitere Verifizierung mittels MRT ist hier i.d.R. nicht erforderlich. Im Falle von muskulären Verletzungen wie Muskelfaserrissen ist der Ultraschall wegen seiner schnellen Verfügbarkeit und guten Auflösung im Nahbereich Methode der Wahl [16]. Im Bereich des muskulotendinösen Übergangs ist hier jedoch das MRT sensitiver [5, 8,9].

Weitere Vorteile des Ultraschalls liegen in der schnellen Verfügbarkeit (z.T. bereits am Spielfeldrand bzw. der Umkleidekabine mit mobilen Geräten), den geringen Kosten und der Möglichkeit der dynamischen Untersuchung (z.B. Sehnenrupturen oder Gelenkinstabilität bei ligamentärer Partialruptur), welche die Sensitivität des Verfahrens nochmals erhöht [12]. Von Nachteil ist hier aber auch die hohe Untersucherabhängigkeit und somit auch z.T. geringe Reproduzierbarkeit zu nennen.
Abb. 1 Beidseitiges suprapopliteales Hämatom nach beidseitigem proximalem Abriss der ischiokruralen Muskulatur.

\section{Röntgen}

Bei der Diagnostik von Frakturen bleibt das Nativröntgen das Diagnostikum der Wahl und ist i.d.R. immer unmittelbar verfügbar. Auch beim Verdacht auf eine rein ligamentäre Verletzung im Gelenkbereich sollte ebenfalls immer eine Röntgenaufnahme erfolgen. Diese kann begleitende Frakturen ausschließen oder bei knöchernen Bandausrissen (Abb.4) die Diagnose bereits erhärten. Gehaltene Aufnahmen, welche in der Vergangenheit gerne bei ligamentären Verletzungen am Knie- oder Sprunggelenk angewendet worden sind, gehören heutzutage nicht mehr zur Akutdiagnostik. Aufgrund der schmerzhaften Technik für den Patienten und der eingeschränkten Aussagekraft bei schmerzbedingtem Gegenspannen sind sie den chronischen Verläufen vorbehalten [3] und in der Akutdiagnostik obsolet.

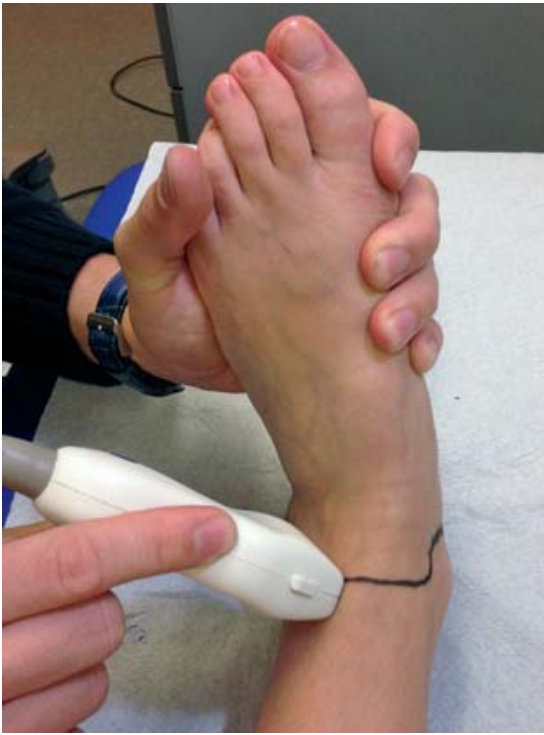

Abb. 2 Dynamische Untersuchung des vorderen Syndesmosenanteils (AITFL) nach Distorsionstrauma des oberen Sprunggelenks.

\section{Magnetresonanztomografie (MRT)}

Beim Verdacht auf ligamentäre Verletzungen, insbesondere mit klinisch hochgradiger Instabilität, ist das MRT derzeit der Goldstandard. Viele Verletzungen können mit hoher Sensitivität dargestellt werden, wobei die Spezifität z.T. eher eingeschränkt ist. Die hierdurch bedingte starke Frequentierung dieser Untersuchungstechnik führt jedoch zu teilweise erheblichen Wartezeiten von Wochen bis Monaten, was ihre Praktikabilität einschränkt.

Somit ist, wie bereits zuvor erwähnt, anhand einer genauen klinischen Untersuchung die Notwendigkeit und Dringlichkeit eines MRT bereits im Vorfeld zu klären.
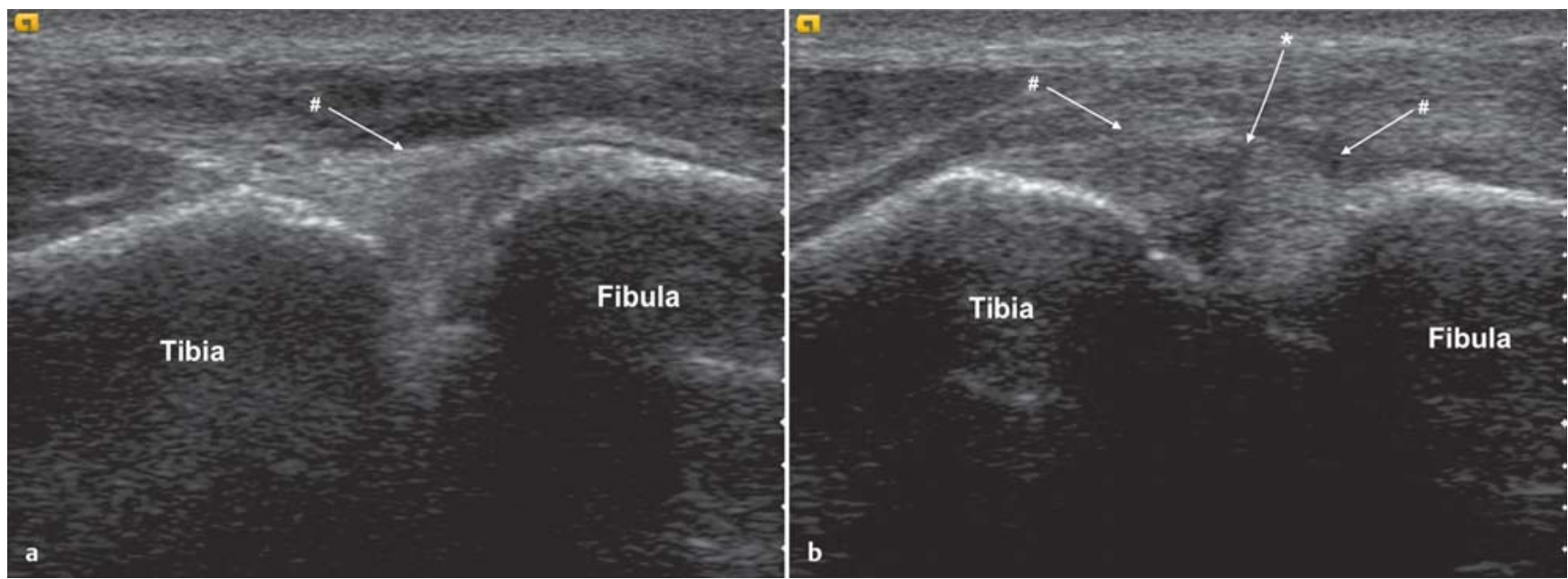

Abb. $\mathbf{3}$ a und b Sonografie des ATFL (\#): a intakter Zustand. b zentrale transligamentäre Ruptur ( ${ }^{*}$ ) mit Erweiterung des tibiofibularen Abstands in der dynamischen Untersuchung. 


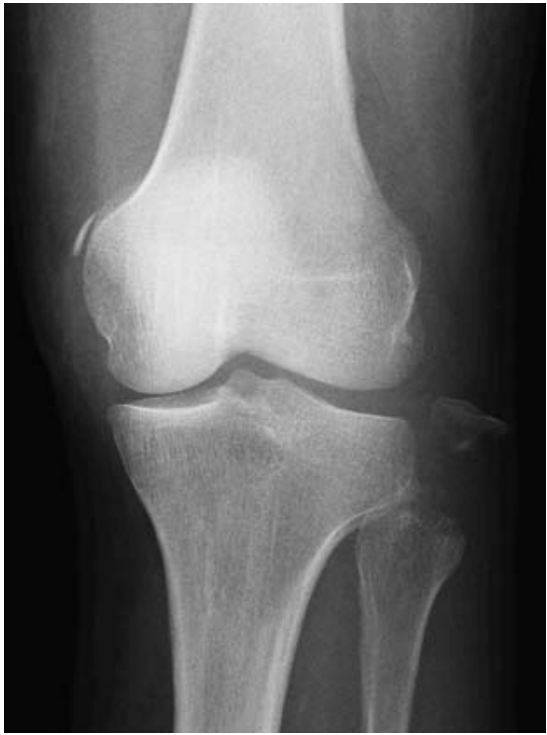

Abb. 4 Fibularer knöcherner Ausriss des lateralen Kollateralbands.

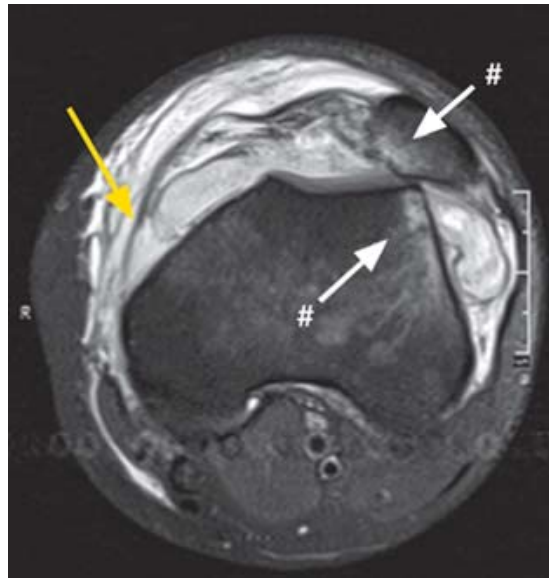

Abb. 6 Femoraler Abriss des medialen patel lofemoralen Ligaments (MPFL, gelber Pfeil). Bone Bruise patellar und laterale Trochlea (\#). Knorpelläsion retropatellar (MRT).

Nur wenige Diagnosen erfordern eine unmittelbare kernspintomografische Untersuchung. Bei der Darstellung von Sehnenrupturen erzielt das MRT zwar eine höhere Sensitivität und Spezifität als der Ultraschall, ist i.d.R. aber als additives Verfahren nicht notwendig [1,14]. In der Diagnostik der hinteren Kreuzbandruptur (HKB; Abb. 5) oder der frischen Patellaluxation (Abb.6) ist sie jedoch unumgänglich und innerhalb weniger Tage durchzuführen. Bei der frischen HKBRuptur kann der frühzeitige Beginn einer konservativen Behandlung mit adäquater Orthesenversorgung zu hervorragenden Ergebnissen und eine später notwendig HKB-Ersatzplastik verhindern. Bei der frischen Patellaluxation ist das

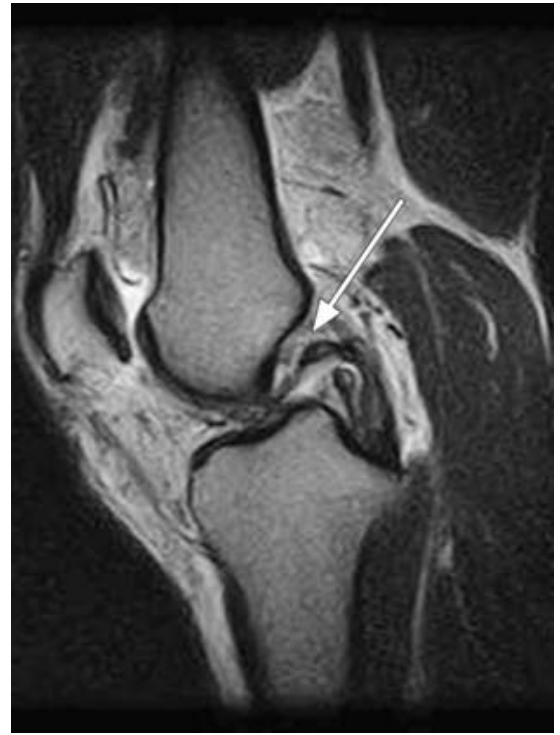

Abb. 5 Proximale Ruptur des hinteren Kreuzbands (weißer Pfeil) im MRT.

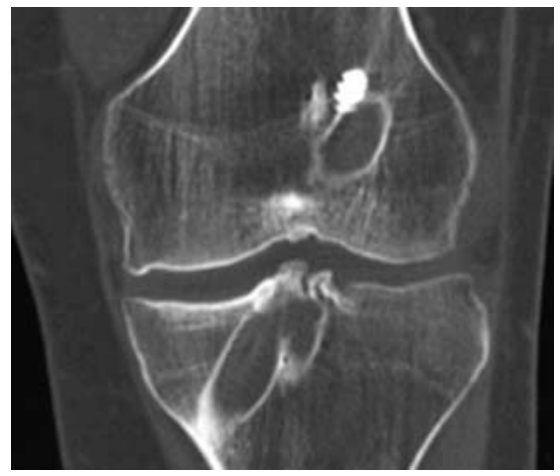

Abb.7 CT zur Darstellung der Bohrkanäle bei Ruptur einer vorderen Kreuzbandersatzplastik.

MRT zur OP-Planung mit Lokalisation der Ruptur des medialen patellofemoralen Ligaments (MPFL) und begleitender chondraler Läsionen essenziell.

\section{Computertomografie}

Das CT nimmt den geringsten Stellenwert in der Diagnostik der Sportverletzungen ein. Selbst bei knöchernen Bandausrissen oder kleineren artikulären Impressionsfrakturen lässt sich der Frakturverlauf mit der aktuellen Qualität der MR-Tomografie ausreichend genau darstellen. Eine mögliche Indikation sind Rerupturen von ligamentären Ersatzplastiken zur Darstellung der ehemaligen Bohrkanäle (Abb. 7) oder bei größeren Impressionsfrakturen.

\section{Schlussfolgerung}

Bei Sportverletzungen gilt selbiges wie in der restlichen Medizin. Anamnese und klinische Untersuchung sind der Grundstein der Diagnostik. Aus den hieraus erhobenen Befunden ergibt sich der Algorithmus und die Dringlichkeit für die weitere apparative Diagnostik. Trotz des Vormarsches des MRT mit seiner vielfach hohen diagnostischen Aussagekraft sollten Basisdiagnostika wie der Ultraschall nicht in Vergessenheit geraten. Auch mit diesem können viele Verletzungen im Sportbereich suffizient diagnostiziert werden und somit eine Verzögerung der Therapieeinleitung reduziert werden.

\section{Literatur}

${ }^{1}$ Aström M, Gentz CF, Nilsson P et al. Imaging in chronic achilles tendinopathy: a comparison of ultrasonography, magnetic resonance imaging and surgical findings in 27 histologically verified cases. Skeletal Radiol 1996; 25: 615-620

2 Belli P, Costantini M, Mirk P et al. Sonographic diagnosis of distal biceps tendon rupture: a prospective study of 25 cases. J Ultrasound Med 2001; 20: 587-595

3 Best R, Mauch F, Bauer G. [Evidence for treatment of acute syndesmosis injuries in sports]. Unfallchirurg 2013; 116: 504-511

4 Bianchi S, Zwass A, Abdelwahab IF et al. Diagnosis of tears of the quadriceps tendon of the knee: value of sonography. AJR Am J Roentgenol 1994; 162: 1137-1140

5 De Smet AA, Best TM. MR imaging of the distribution and location of acute hamstring injuries in athletes. AJR Am J Roentgenol 2000; 174: 393-399

${ }^{6}$ Henari S, Banks LN, Radovanovic I et al. Ultrasonography as a diagnostic tool in assessing deltoid ligament injury in supination external rotation fractures of the ankle. Orthopedics 2011; 34: e639-643

${ }^{7}$ Ilan DI, Tejwani N, Keschner $M$ et al. Quadriceps tendon rupture. J Am Acad Orthop Surg 2003; 11: 192-200

8 Järvinen MJ, Lehto MU. The effects of early mobilisation and immobilisation on the healing process following muscle injuries. Sports Med 1993; 15: 78-89

${ }^{9}$ Kieb $M$, Lorbach O, Engelhardt $M$. [Muscle injuries: diagnostics and treatments]. Orthopade 2010; 39: 1098-1107

10 LaRocco BG, Zlupko G, Sierzenski P. Ultrasound diagnosis of quadriceps tendon rupture. J Emerg Med 2008; 35: 293-295

11 Margetić P, Salaj M, Lubina IZ. The Value of Ultrasound in Acute Ankle Injury: Comparison With MR. Eur J Trauma Emerg Surg 2008; 35: 141-146

12 Mei-Dan O, Kots E, Barchilon Vet al. A dynamic ultrasound examination for the diagnosis of ankle syndesmotic injury in professional athletes: a preliminary study. Am J Sports Med 2009; 37: 1009-1016

13 Milz P, Milz S, Steinborn M et al. Lateral ankle ligaments and tibiofibular syndesmosis. 13$\mathrm{MHz}$ high-frequency sonography and MRI compared in 20 patients. Acta Orthop Scand 1998; 69: 51-55

14 Pagenstert G, Leumann A, Frigg $A$ et al. [Achilles tendon ruptures and tibialis anteri- 
or tendon ruptures]. Orthopade 2010; 39: 1135-1147

15 Pijnenburg AC, Glas AS, De Roos MA et al. Radiography in acute ankle injuries: the Ottawa Ankle Rules versus local diagnostic decision rules. Ann Emerg Med 2002; 39: 599-604
16 Smith J, Finnoff JT. Diagnostic and interventional musculoskeletal ultrasound: part 2 . Clinical applications. PM R 2009; 1: 162-177

17 Thermann $H$, Hüfner $T$, Tscherne $H$. [Achilles tendon rupture]. Orthopade 2000; 29: 235250
Dr. med. Björn H. Drews

Funktionsoberarzt

Klinik für Unfall-, Hand-, Plastische und Wiederherstellungschirurgie Zentrum für Chirurgie Universitätsklinikum Ulm Albert-Einstein-Allee 23 $89081 \mathrm{Ulm}$

bjoern.drews@uniklinik-ulm.de 